\title{
Some Retarded Difference Inequalities of Product Form and Their Application
}

\author{
Zongyi Hou, ${ }^{1}$ Shanhe $\mathrm{Wu}^{2}$, and Wu-Sheng Wang ${ }^{1}$ \\ ${ }^{1}$ School of Mathematics and Statistics, Hechi University, Yizhou, Guangxi 546300, China \\ ${ }^{2}$ Department of Mathematics and Computer Science, Longyan University, Longyan, Fujian 364012, China
}

Correspondence should be addressed to Shanhe Wu; shanhewu@gmail.com

Received 18 February 2014; Accepted 8 July 2014; Published 22 July 2014

Academic Editor: Weinian Zhang

Copyright (c) 2014 Zongyi Hou et al. This is an open access article distributed under the Creative Commons Attribution License, which permits unrestricted use, distribution, and reproduction in any medium, provided the original work is properly cited.

A class of new nonlinear retarded difference inequalities is established. An application of the obtained inequalities to the estimation of finite difference equations is given.

\section{Introduction}

Difference inequalities which give explicit bounds on unknown functions provide a very useful and important tool in the study of many qualitative as well as quantitative properties of solutions of nonlinear difference equations. Various investigators have discovered many useful and new difference inequalities, mainly inspired by their applications in various branches of difference equations; see [1-25] and the references cited therein.

Sugiyama [2] established the most precise and complete discrete analogue of the Gronwall inequality (see [1]) in the following form.

Let $u(n)$ and $f(n)$ be nonnegative functions defined for $n \in \mathbf{N}$, and suppose that $f(n) \geq 0$ for every $n \in \mathbf{N}$. If

$$
u(n) \leq u_{0}+\sum_{s=n_{0}}^{n-1} f(s) u(s), \quad n \in \mathbf{N},
$$

where $\mathbf{N}$ is the set of points $n_{0}+k(k=0,1,2, \ldots), n_{0} \geq 0$ is a given integer, and $u_{0}$ is a nonnegative constant, then

$$
u(n) \leq u_{0} \prod_{s=n_{0}}^{n-1}[1+f(s)], \quad n \in \mathbf{N} .
$$

Pachpatte [4] established a generalized discrete analogue of the Gronwall inequality in the following form.
Let $m(s)$ be a positive and monotone nondecreasing function on $\mathbf{N}$, and let $a(s), b(s)$ be nonnegative functions on $\mathbf{N}$. If $u(n)$ satisfies

$$
u(n) \leq m(s)+\sum_{s=n_{0}}^{n-1} a(s)\left(u(s)+\sum_{\tau=n_{0}}^{s-1} b(\tau) u(\tau)\right), \quad \forall n \in \mathbf{N}
$$

then

$$
u(n) \leq P(n) m(s), \quad \forall n \in \mathbf{N},
$$

where

$$
P(n)=1+\sum_{s=n_{0}}^{n-1} a(s) \prod_{\tau=n_{0}}^{s-1}[1+a(\tau)+b(\tau)], \quad \forall n \in \mathbf{N} .
$$

Besides the results mentioned above, the following results are closely related to the investigation of the present paper, and, particularly, they will be used as lemmas for the proofs of our main results in Theorems 3 and 4 .

Lemma 1 (see [5]). Let $u(n), a(n), b(n), c(n)$, and $d(n)$ be nonnegative functions defined on $\mathbf{N}$, for which the inequality

$$
\begin{aligned}
u(n) \leq & u_{0}+\sum_{s=n_{0}}^{n-1} a(s) u(s)+\sum_{s=n_{0}}^{n-1} b(s) \\
& \times\left(\sum_{t=n_{0}}^{s-1} c(t)\left(\sum_{\tau=n_{0}}^{t-1} d(\tau) u^{\alpha}(\tau)\right)\right), \quad \forall n \in \mathbf{N},
\end{aligned}
$$


holds, where $u_{0}$ is a nonnegative constant and $0<\alpha<1$. If $1+a(n)-b(n) \geq 0$ and $1+a(n)+b(n)-c(n) \geq 0$ for all $n \in \mathbf{N}$, then

$$
\begin{aligned}
u(n) \leq & u_{0} \prod_{s=n_{0}}^{n-1}[1+a(s)-b(s)] \\
+ & \sum_{s=n_{0}}^{n-1} b(s) \prod_{t=s+1}^{n-1}[1+a(t)-b(t)] \\
\times & \left\{u_{0} \prod_{t=n_{0}}^{s-1}[1+a(t)+b(t)-c(t)]\right. \\
& +\sum_{t=n_{0}}^{s-1} c(t) \prod_{\tau=t+1}^{s-1}[1+a(\tau)+b(\tau)-c(\tau)] \\
& \times \prod_{\tau=n_{0}}^{t-1}[1+a(\tau)+b(\tau)+c(\tau)] \\
& \times\left[u_{0}^{1-\alpha}+(1-\alpha) \sum_{\tau=n_{0}}^{t-1} d(\tau)\right. \\
& \times \prod_{\rho=n_{0}}^{\tau}[1+a(\rho)+b(\rho)
\end{aligned}
$$

Lemma 2 (see $[3,7])$. Let $w(n, r)$ be a real-valued function defined for $n \in \mathbf{N}, 0 \leq r<\infty$ and monotone nondecreasing with respect to $r$ for any fixed $n \in \mathbf{N}$. Let $u(n)$ be a real-valued function defined for $n \in \mathbf{N}$ such that

$$
\Delta u(n) \leq w(n, u(n)), \quad \forall n \in \mathbf{N}
$$

Let $r(n)$ be a solution of

$$
\Delta r(n)=w(n, r(n)), \quad r\left(n_{0}\right)=r_{0}, \quad \forall n \in \mathbf{N},
$$

such that $u\left(n_{0}\right) \leq r\left(n_{0}\right)$. Then

$$
u(n) \leq r(n), \quad \forall n \in \mathbf{N}
$$

Pachpatte $[7,8]$ also established some difference inequalities of product form as follows.

Let $u, a$, and $b$ be nonnegative functions defined on $\mathbf{N}$ and let $c$ be a nonnegative constant. Let $w(n, r)$ be a nonnegative function defined for $n \in \mathbf{N}, 0 \leq r<\infty$ and monotone nondecreasing with respect to $r$ for any fixed $n \in \mathbf{N}$. If $u(n)$ satisfies

$$
\begin{aligned}
u^{2}(n) \leq & c^{2}+2 \sum_{s=n_{0}}^{n-1} u(s) \\
\times & {\left[a(s)\left(u(s)+\sum_{t=n_{0}}^{s-1} b(t) u(t)\right)\right.} \\
& +w(s, u(s))], \quad \forall n \in \mathbf{N},
\end{aligned}
$$

then

$$
u(n) \leq P(n) r(n), \quad \forall n \in \mathbf{N},
$$

where $P(n)$ is defined by (5) and $r(n)$ is a solution of

$$
\Delta r(n)=w(n, P(n) r(n)), \quad r\left(n_{0}\right)=c, \quad \forall n \in \mathbf{N} .
$$

Let $u, a$, and $b$ be nonnegative functions defined for $n \in \mathbf{N}$ and let $c$ be a nonnegative constant. Let $w(n, r)$ be a nonnegative function defined for $n \in \mathbf{N}, 0 \leq r<\infty$ and monotone nondecreasing with respect to $r$ for any fixed $n \in \mathbf{N}$. If $u(n)$ satisfies

$$
\begin{aligned}
& u^{2}(n) \leq c^{2}+\sum_{s=n_{0}}^{n-1} a(s)(u(s+1)+u(s)) \\
& \times\left[\left(u(s)+\sum_{\tau=n_{0}}^{s-1} b(\tau) u(\tau)\right)+w(s, u(s))\right], \\
& \forall n \in \mathbf{N},
\end{aligned}
$$

then

$$
u(n) \leq P(n) r(n), \quad \forall n \in \mathbf{N},
$$

where $P(n)$ is defined by (5) and $r(n)$ is a solution of the difference equation

$$
\Delta r(n)=a(n) w(n, P(n) r(n)), \quad r\left(n_{0}\right)=c, \quad \forall n \in \mathbf{N} .
$$

Motivated by the results given in $[5,7,8]$, in this paper, we discuss new nonlinear difference inequalities:

$u^{2}(n)$

$$
\begin{aligned}
& \leq c^{2}+\sum_{s=n_{0}}^{n-1} f(s)(u(s+1)+u(s)) \\
& \times\left[\left(u(s)+\sum_{t=n_{0}}^{s-1} g(t) \sum_{\tau=n_{0}}^{t-1} h(\tau) u^{\alpha}(\tau)\right)+w(s, u(s))\right], \\
& \quad 0<\alpha<1, \quad \forall n \in \mathbf{N} .
\end{aligned}
$$

It is important to note that the inequality given above can be used as tools in the study of certain classes of finite difference equations. In Section 3 we provide an application of our results to the estimation of finite difference equations. 


\section{Main Results}

Throughout this paper, let $\mathbf{N}:=\left\{n_{0}, n_{0}+1, n_{0}+2, \ldots\right\}$ and $\mathbf{N}_{T}:=\left\{n_{0}, n_{0}+1, n_{0}+2, \ldots, T\right\}, T \in \mathbf{N}$. For function $u(n), n \in \mathbf{N}$, we define the operator $\Delta$ by $\Delta u(n)=$ $u(n+1)-u(n)$. Obviously, the linear difference equation $\Delta u(n)=f(n)$ with the initial condition $u\left(n_{0}\right)=0$ has the solution $u(n)=\sum_{s=n_{0}}^{n-1} f(s)$. For convenience, in the sequel we complementarily define that $\sum_{s=n_{0}}^{n_{0}-1} f(s)=0$ and $\prod_{s=n_{0}}^{n_{0}-1} f(s)=$ 1.

Theorem 3. Let $m(s)$ be a nonnegative and monotone nondecreasing function defined on $\mathbf{N}$, and let $f(s), g(s)$, and $h(s)$ be nonnegative functions defined on $\mathbf{N}_{0}$. Let $\alpha$ be a constant with $0<\alpha<1$. If $u(n)$ satisfies

$$
\begin{aligned}
u(n) \leq & m(n) \\
& +\sum_{s=n_{0}}^{n-1} f(s) u(s)+\sum_{s=n_{0}}^{n-1} f(s) \\
& \times \sum_{t=n_{0}}^{s-1} g(t)\left(\sum_{\tau=n_{0}}^{t-1} h(\tau) u^{\alpha}(\tau)\right), \quad \forall n \in \mathbf{N},
\end{aligned}
$$

then

$$
u(n) \leq W_{1}(m(n), n), \quad \forall n \in \mathbf{N},
$$

where

$$
W_{1}(m(n), n)
$$$$
:=m(n)+\sum_{s=n_{0}}^{n-1} f(s)
$$$$
\times\left\{m(n) \prod_{t=n_{0}}^{s-1}[1+2 f(t)-g(t)]\right.
$$$$
+\sum_{t=n_{0}}^{s-1} g(t) \prod_{\tau=t+1}^{s-1}[1+2 f(\tau)-g(\tau)]
$$$$
\times \prod_{\tau=n_{0}}^{t-1}[1+2 f(\tau)+g(\tau)]
$$$$
\times\left[m^{1-\alpha}(n)+(1-\alpha) \sum_{\tau=n_{0}}^{t-1} h(\tau)\right.
$$$$
\left.\left.\times \prod_{\rho=n_{0}}^{\tau}[1+2 f(\rho)+g(\rho)]^{\alpha-1}\right]^{1 /(1-\alpha)}\right\}, \quad \forall n \in \mathbf{N} .
$$

Proof. Fix $T \in \mathbf{N}$, where $T$ is chosen arbitrarily; since $m(t)$ is a nonnegative and monotone nondecreasing function, from (18), we have

$$
\begin{array}{r}
u(n) \leq m(T)+\sum_{s=n_{0}}^{n-1} f(s) u(s) \\
+\sum_{s=n_{0}}^{n-1} f(s) \sum_{t=n_{0}}^{s-1} g(t)\left(\sum_{\tau=n_{0}}^{t-1} h(\tau) u^{\alpha}(\tau)\right), \\
\forall n \in \mathbf{N}_{T} .
\end{array}
$$

Now an application of Lemma 1 to (21) yields

$$
\begin{aligned}
u(n) \leq & m(T)+\sum_{s=n_{0}}^{n-1} f(s) \\
\times & \left\{m(T) \prod_{t=n_{0}}^{s-1}[1+2 f(t)-g(t)]\right. \\
& +\sum_{t=n_{0}}^{s-1} g(t) \prod_{\tau=t+1}^{s-1}[1+2 f(\tau)-g(\tau)] \\
& \times \prod_{\tau=n_{0}}^{t-1}[1+2 f(\tau)+g(\tau)]
\end{aligned}
$$$$
\times\left[m^{1-\alpha}(T)+(1-\alpha) \sum_{\tau=n_{0}}^{t-1} h(\tau)\right.
$$$$
\left.\left.\times \prod_{\rho=n_{0}}^{\tau}[1+2 f(\rho)+g(\rho)]^{\alpha-1}\right]^{1 /(1-\alpha)}\right\},
$$$$
\forall n \in \mathbf{N}_{T} .
$$

Since $T \in \mathbf{N}$ is arbitrary, from (22), we get the required estimate (19).

Theorem 4. Let $u, f, g$, and $h$ be nonnegative functions defined for $n \in \mathbf{N}$ and let $c$ be a nonnegative constant. Let $w(n, r)$ be a real-valued function defined for $n \in \mathbf{N}, 0 \leq r<\infty$ and monotone nondecreasing with respect to $r$ for any fixed $n \in \mathbf{N}$. Let $\alpha$ be a constant with $0<\alpha<1$. If $u(n)$ satisfies (17), then

$$
u(n) \leq W_{1}(v(n), n), \quad \forall n \in \mathbf{N},
$$

where $W_{1}(v(n), n)$ is defined by (20) in Theorem 3 and $v(n)$ is a solution of the difference equation

$$
\begin{array}{r}
\Delta r(n)=f(n) w\left(n, W_{1}(r(n), n)\right), \quad r\left(n_{0}\right)=c, \\
\forall n \in \mathbf{N} .
\end{array}
$$

Proof. We first assume that $c>0$ and define a function $z(n)$ by the right-hand side of (17). Then $z(n)$ is a nonnegative and monotone nondecreasing function defined on $\mathbf{N}_{0}$. We have

$$
z\left(n_{0}\right)=c^{2}, \quad u(n) \leq \sqrt{z(n)}, \quad \forall n \in \mathbf{N} .
$$


Using the definitions of the operator $\Delta$ and $z$, we obtain

$$
\begin{aligned}
& \Delta z(n) \\
& =f(n)(u(n+1)+u(n)) \\
& \times\left[\left(u(n)+\sum_{t=n_{0}}^{n-1} g(t) \sum_{\tau=n_{0}}^{t-1} h(\tau) u^{\alpha}(\tau)\right)+w(n, u(n))\right] \\
& \leq f(n)(\sqrt{z(n+1)}+\sqrt{z(n)}) \\
& \times\left[\left(\sqrt{z(n)}+\sum_{t=n_{0}}^{n-1} g(t) \sum_{\tau=n_{0}}^{t-1} h(\tau)(\sqrt{z(\tau)})^{\alpha}\right)\right. \\
& \quad+w(n, \sqrt{z(n)})], \quad \forall n \in \mathbf{N} .
\end{aligned}
$$

From (26), we have

$$
\begin{aligned}
& \Delta(\sqrt{z(n)}) \\
& =\frac{\Delta z(n)}{\sqrt{z(n+1)}+\sqrt{z(n)}} \\
& \leq f(n)\left[\left(\sqrt{z(n)}+\sum_{t=n_{0}}^{n-1} g(t)\right.\right. \\
& \left.\left.\quad \times \sum_{\tau=n_{0}}^{t-1} h(\tau)(\sqrt{z(\tau)})^{\alpha}\right)+w(n, \sqrt{z(n)})\right], \\
& \quad \forall n \in \mathbf{N} .
\end{aligned}
$$

Setting $n=s$ in (27) and substituting $s=n_{0}, n_{0}+1, n_{0}+$ $2, \ldots, n-1$, successively, we get

$$
\begin{aligned}
\sqrt{z(n)} \leq & c+\sum_{s=n_{0}}^{n-1} f(s) \\
\times & {\left[\left(\sqrt{z(s)}+\sum_{t=n_{0}}^{s-1} g(t) \sum_{\tau=n_{0}}^{t-1} h(\tau)(\sqrt{z(\tau)})^{\alpha}\right)\right.} \\
& +w(s, \sqrt{z(s)})], \quad \forall n \in \mathbf{N} .
\end{aligned}
$$

Define a function $z_{1}(n)$ by

$$
z_{1}(n)=c+\sum_{s=n_{0}}^{n-1} f(s) w(s, \sqrt{z(s)}), \quad \forall n \in \mathbf{N}
$$

Then $z_{1}\left(n_{0}\right)=c$ and

$$
\Delta z_{1}(n)=f(n) w(n, \sqrt{z(n)}), \quad \forall n \in \mathbf{N} .
$$

Using (29), inequality (28) can be written as

$$
\begin{aligned}
\sqrt{z(n)} \leq & z_{1}(n) \\
& +\sum_{s=n_{0}}^{n-1} f(s)\left(\sqrt{z(s)}+\sum_{t=n_{0}}^{s-1} g(t) \sum_{\tau=n_{0}}^{t-1} h(\tau)\right. \\
& \left.\times(\sqrt{z(\tau)})^{\alpha}\right), \quad \forall n \in \mathbf{N},
\end{aligned}
$$

since $z_{1}(n)$ is positive and monotone nondecreasing for $n \in \mathbf{N}$ and $f(s), g(s)$, and $h(s)$ satisfy the conditions in Theorem 3 . Now an application of Theorem 3 to (31) yields

$$
\sqrt{z(n)} \leq W_{1}\left(z_{1}(n), n\right), \quad \forall n \in \mathbf{N}
$$

where $W_{1}\left(z_{1}(n), n\right)$ is defined by (20) in Theorem 3. Since $w(n, r)$ is monotone nondecreasing with respect to $r$ for any fixed $n \in \mathbf{N}$, from (30) and (32), we have

$$
\Delta z_{1}(n) \leq f(n) w\left(n, W_{1}\left(z_{1}(n), n\right)\right), \quad \forall n \in \mathbf{N}
$$

Now with a suitable application of Lemma 2, we obtain

$$
z_{1}(n) \leq v(n), \quad \forall n \in \mathbf{N}
$$

where $v(n)$ is a solution of (24). Using (25), (32), and (34), we obtain our required estimation (23).

If $c$ is nonnegative, we can carry out the above procedure with $c+\epsilon$ instead of $c$, where $\epsilon$ is an arbitrary small number. Letting $\epsilon \rightarrow 0$, we obtain (23).

\section{Application to Finite Difference Equations}

In this section, we apply our result to the following difference equation:

$$
\begin{aligned}
\Delta x(n)=f(n) & \\
\times & {\left[F\left(n, x(n), \sum_{t=n_{0}}^{n-1} g(t) \sum_{\tau=n_{0}}^{t-1} H(t, \tau, x(\tau))\right)\right.} \\
& +K(n, x(n))], \quad \forall n \in \mathbf{N},
\end{aligned}
$$

where $K, H$, and $F$ are real-valued functions defined, respectively, on $\mathbf{N} \times \mathbf{R}, \mathbf{N}^{2} \times \mathbf{R}$, and $\mathbf{N} \times \mathbf{R}^{2}$ and $f$ is as defined in Theorem 4 . We assume that

$$
\begin{aligned}
|K(n, x(n))| & \leq w(n,|x(n)|), \\
|H(n, t, x(t))| & \leq \sum_{t=n_{0}}^{n-1} h(t)|x(t)|^{\alpha}, \\
|F(n, x(n), y(n))| & \leq|x(n)|+|y(n)|,
\end{aligned}
$$


where $g, h, w$, and $\alpha$ are as defined in Theorem 4. From (35), we have

$$
\begin{aligned}
x^{2}(n+1) & -x^{2}(n) \\
= & f(n)[x(n+1)+x(n)] \\
\times & {\left[F\left(n, x(n), \sum_{t=n_{0}}^{n-1} g(t) \sum_{\tau=n_{0}}^{t-1} H(t, \tau, x(\tau))\right)\right.} \\
& +K(n, x(n))], \quad \forall n \in \mathbf{N} .
\end{aligned}
$$

Using conditions (36), we obtain

$$
\begin{aligned}
|x(n)|^{2}= & x^{2}\left(n_{0}\right)+\sum_{s=n_{0}}^{n-1} f(s)[|x(s+1)|+|x(s)|] \\
\times & {\left[|x(s)|+\sum_{t=n_{0}}^{s-1} g(t) \sum_{\tau=n_{0}}^{t-1} h(\tau)|x(\tau)|^{\alpha}\right.} \\
& +w(s,|x(s)|)], \quad \forall n \in \mathbf{N} .
\end{aligned}
$$

Now an application of Theorem 4 to (39) yields the estimation of the difference equation (35) as follows:

$$
\begin{aligned}
& |x(n)| \\
& \leq v(n) \\
& +\sum_{s=n_{0}}^{n-1} f(s)\left\{v(n) \prod_{t=n_{0}}^{s-1}[1+2 f(t)-g(t)]\right. \\
& +\sum_{t=n_{0}}^{s-1} g(t) \prod_{\tau=t+1}^{s-1}[1+2 f(\tau)-g(\tau)] \\
& \quad \times \prod_{\tau=n_{0}}^{t-1}[1+2 f(\tau)+g(\tau)]
\end{aligned}
$$

$$
\begin{aligned}
& \times\left[v^{1-\alpha}(n)+(1-\alpha) \sum_{\tau=n_{0}}^{t-1} h(\tau)\right. \\
& \left.\left.\quad \times \prod_{\rho=n_{0}}^{\tau}[1+2 f(\rho)+g(\rho)]^{\alpha-1}\right]^{1 /(1-\alpha)}\right\},
\end{aligned}
$$

$\forall n \in \mathbf{N}$,

where $v(n)$ is a solution of the difference equation

$$
\begin{array}{r}
\Delta r(n)=f(n) w\left(n, W_{1}(r(n), n)\right), \quad r\left(n_{0}\right)=\left|x\left(n_{0}\right)\right|, \\
\forall n \in \mathbf{N} .
\end{array}
$$

\section{Conflict of Interests}

The authors declare that there is no conflict of interests regarding the publication of this paper.

\section{Acknowledgments}

The present investigation was supported by the National Natural Science Foundation of China (no. 11161018), the Foundation of Scientific Research Project of Fujian Province Education Department (no. JK2012049), the Guangxi Natural Science Foundation (no. 2012GXNSFAA053009), and the Scientific Research Foundation of the Education Department of Guangxi Zhuang Autonomous Region (no. 201106LX599 and no. 201204LX391). The authors are grateful to the anonymous referees for their careful comments and valuable suggestions on this paper.

\section{References}

[1] T. H. Gronwall, "Note on the derivatives with respect to a parameter of the solutions of a system of differential equations," Annals of Mathematics, vol. 20, no. 4, pp. 292-296, 1919.

[2] S. Sugiyama, "On the stability problems of difference equations," Bulletin of Science and Engineering Research Laboratory: Waseda University, vol. 45, pp. 140-144, 1969.

[3] B. G. Pachpatte, "Finite-difference inequalities and an extension of Lyapunov's method," The Michigan Mathematical Journal, vol. 18, pp. 385-391, 1971.

[4] B. G. Pachpatte, "On some new discrete inequalities and their applications," Proceedings of the National Academy of Sciences, India, vol. 46, no. 4, pp. 255-262, 1976.

[5] B. G. Pachpatte, "On discrete inequalities related to Gronwall's inequality," Proceedings of the Indian Academy of Science, vol. 85, no. 1, pp. 26-40, 1977.

[6] B. G. Pachpatte, "Finite difference inequalities and discrete time control systems," Indian Journal of Pure and Applied Mathematics, vol. 9, no. 12, pp. 1282-1290, 1978.

[7] B. G. Pachpatte, "Comparison theorems related to a certain inequality used in the theory of differential equations," Soochow Journal of Mathematics, vol. 22, no. 3, pp. 383-394, 1996.

[8] B. G. Pachpatte, "Inequalities applicable in the theory of finite difference equations," Journal of Mathematical Analysis and Applications, vol. 222, no. 2, pp. 438-459, 1998. 
[9] E. Yang, "A new nonlinear discrete inequality and its application," Annals of Differential Equations, vol. 17, no. 3, pp. 261-267, 2001.

[10] B. G. Pachpatte, "On some fundamental integral inequalities and their discrete analogues," Journal of Inequalities in Pure and Applied Mathematics, vol. 2, no. 2, article 15, 2001.

[11] F. W. Meng and W. N. Li, "On some new nonlinear discrete inequalities and their applications," Journal of Computational and Applied Mathematics, vol. 158, no. 2, pp. 407-417, 2003.

[12] W. Cheung and J. Ren, "Discrete non-linear inequalities and applications to boundary value problems," Journal of Mathematical Analysis and Applications, vol. 319, no. 2, pp. 708-724, 2006.

[13] B. G. Pachpatte, Integral and Finite Difference Inequalities and Applications, vol. 205 of North-Holland Mathematics Studies, Elsevier Science, Amsterdam, The Netherlands, 2006.

[14] W. Sheng and W. N. Li, "Bounds on certain nonlinear discrete inequalities," Journal of Mathematical Inequalities, vol. 2, no. 2, pp. 279-286, 2008.

[15] Q. Ma and W. Cheung, "Some new nonlinear difference inequalities and their applications," Journal of Computational and Applied Mathematics, vol. 202, no. 2, pp. 339-351, 2007.

[16] Y. J. Cho, S. S. Dragomir, and Y.-H. Kim, "On some integral inequalities with iterated integrals," Journal of the Korean Mathematical Society, vol. 43, no. 3, pp. 563-578, 2006.

[17] W. S. Wang, "A generalized sum-difference inequality and applications to partial difference equations," Advances in Difference Equations, vol. 2008, Article ID 695495, 12 pages, 2008.

[18] W. Wang, "Estimation on certain nonlinear discrete inequality and applications to boundary value problem," Advances in Difference Equations, vol. 2009, Article ID 708587, 8 pages, 2009.

[19] K. Zheng, S. Zhong, and M. Ye, "Discrete nonlinear inequalities in time control systems," in Proceedings of the International Conference on Apperceiving Computing and Intelligence Analysis (ICACIA '09), pp. 403-406, Chengdu, China, October 2009.

[20] S. Deng, "Nonlinear discrete inequalities with two variables and their applications," Applied Mathematics and Computation, vol. 217, no. 5, pp. 2217-2225, 2010.

[21] W.-S. Wang, Z. Li, and W.-S. Cheung, "Some new nonlinear retarded sum-difference inequalities with applications," Advances in Difference Equations, vol. 2011, article 41, no. 1, 2011.

[22] H. Zhou, D. Huang, W. Wang, and J. Xu, "Some new difference inequalities and an application to discrete-time control systems," Journal of Applied Mathematics, vol. 2012, Article ID 214609, 14 pages, 2012.

[23] Q. He, T. Sun, and H. Xi, "Dynamics of a family of nonlinear delay difference equations," Abstract and Applied Analysis, vol. 2013, Article ID 456530, 4 pages, 2013.

[24] Z.-X. Chen and K. H. Shon, "Fixed points of meromorphic solutions for some difference equations," Abstract and Applied Analysis, vol. 2013, Article ID 496096, 7 pages, 2013.

[25] W. Wang and S. Wu, "Some difference inequalities for iterated sums with applications," Abstract and Applied Analysis, vol. 2013, Article ID 804152, 9 pages, 2013. 


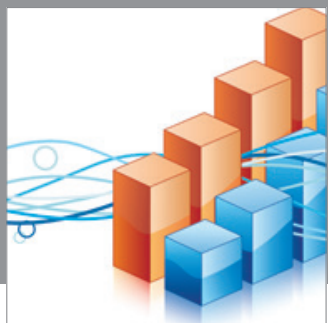

Advances in

Operations Research

mansans

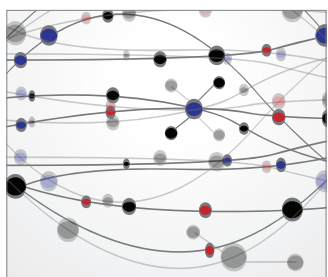

The Scientific World Journal
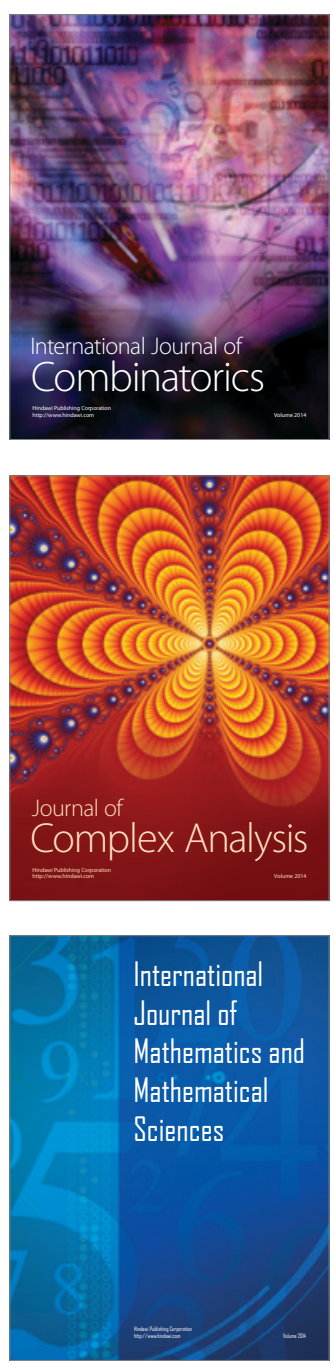
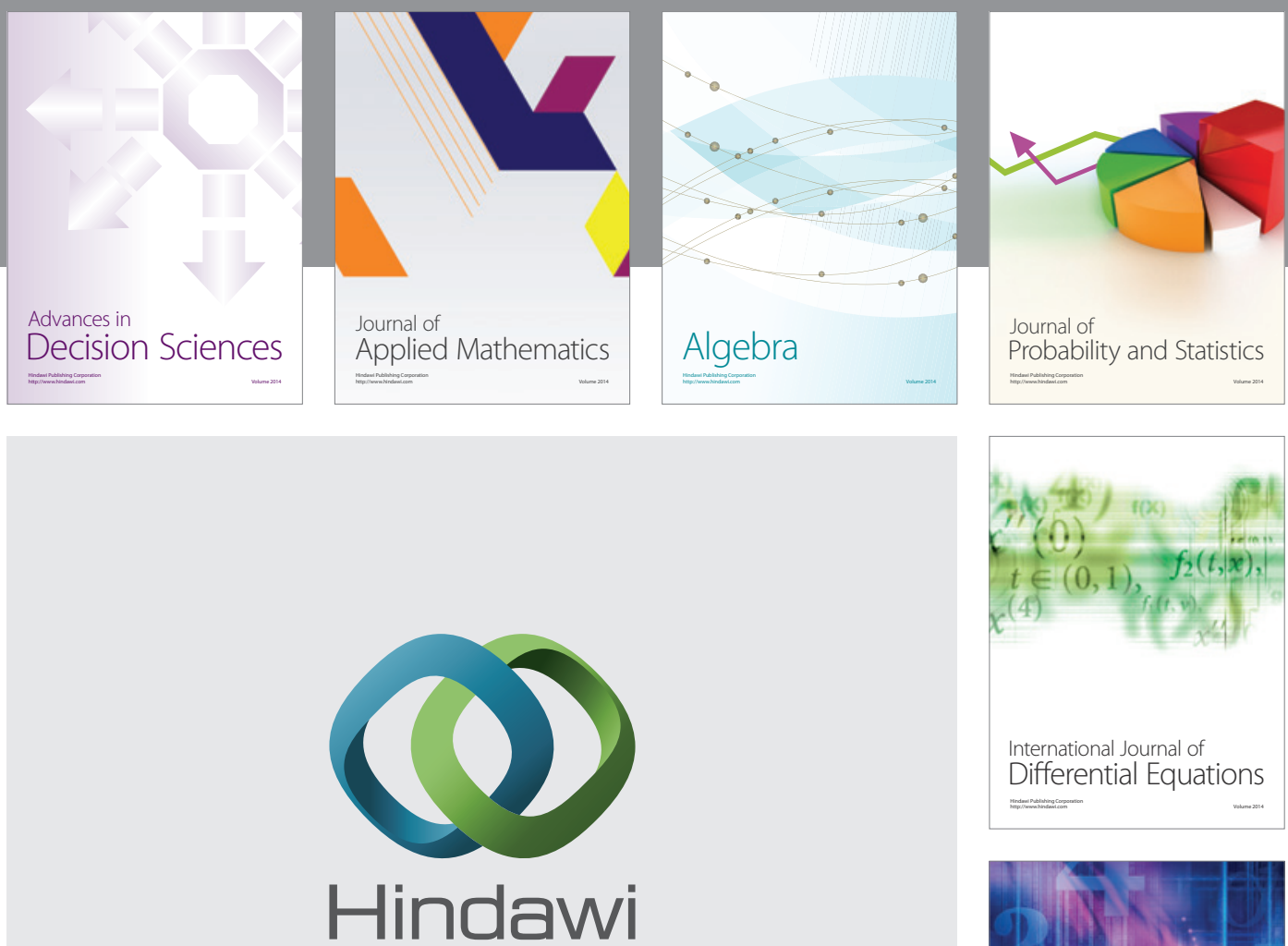

Submit your manuscripts at http://www.hindawi.com
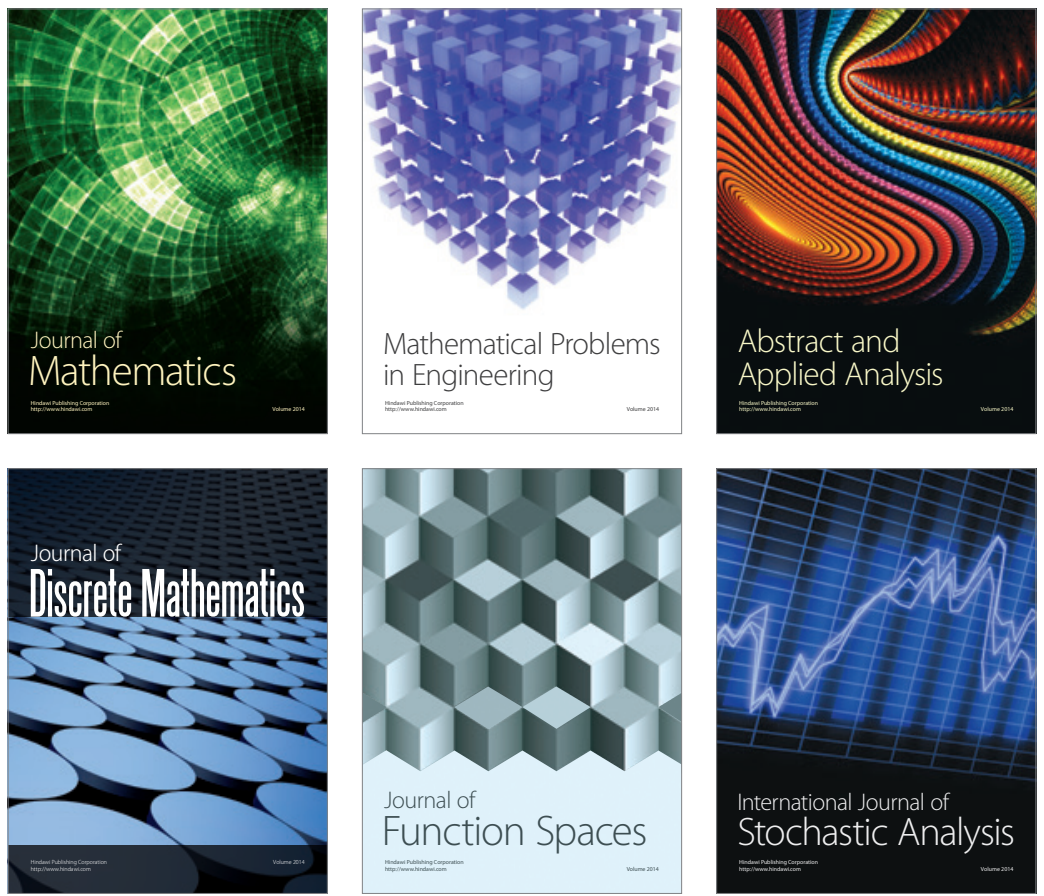

Journal of

Function Spaces

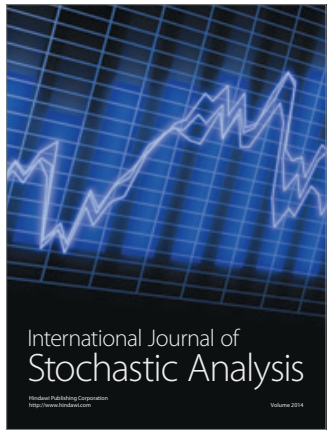

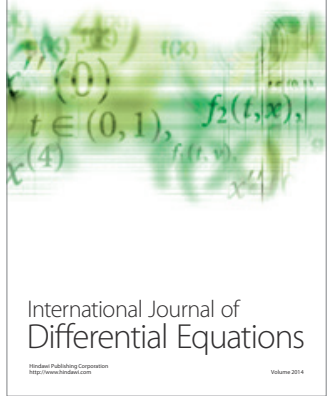
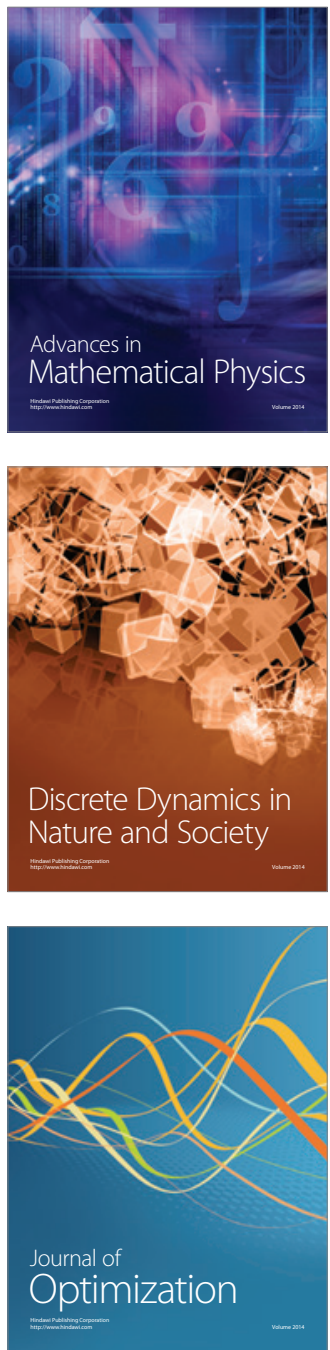